\title{
A versatile atomic force microscope integrated with a scanning electron microscope
}

J. Kreith, T. Strunz, E. J. Fantner, G. E. Fantner, and M. J. Cordill

Citation: Review of Scientific Instruments 88, 053704 (2017); doi: 10.1063/1.4983317

View online: https://doi.org/10.1063/1.4983317

View Table of Contents: http://aip.scitation.org/toc/rsi/88/5

Published by the American Institute of Physics

\section{Articles you may be interested in}

High-throughput atomic force microscopes operating in parallel

Review of Scientific Instruments 88, 033703 (2017); 10.1063/1.4978285

Two-probe atomic-force microscope manipulator and its applications

Review of Scientific Instruments 88, 063701 (2017); 10.1063/1.4985006

Correlative imaging across microscopy platforms using the fast and accurate relocation of microscopic experimental regions (FARMER) method

Review of Scientific Instruments 88, 053702 (2017); 10.1063/1.4982818

Magnetic resonance imaging of granular materials

Review of Scientific Instruments 88, 051806 (2017); 10.1063/1.4983135

Invited Review Article: Tip modification methods for tip-enhanced Raman spectroscopy (TERS) and colloidal probe technique: A 10 year update $(2006-2016)$ review

Review of Scientific Instruments 88, 031101 (2017); 10.1063/1.4978929

Digitally controlled analog proportional-integral-derivative (PID) controller for high-speed scanning probe microscopy

Review of Scientific Instruments 88, 123712 (2017); 10.1063/1.5010181

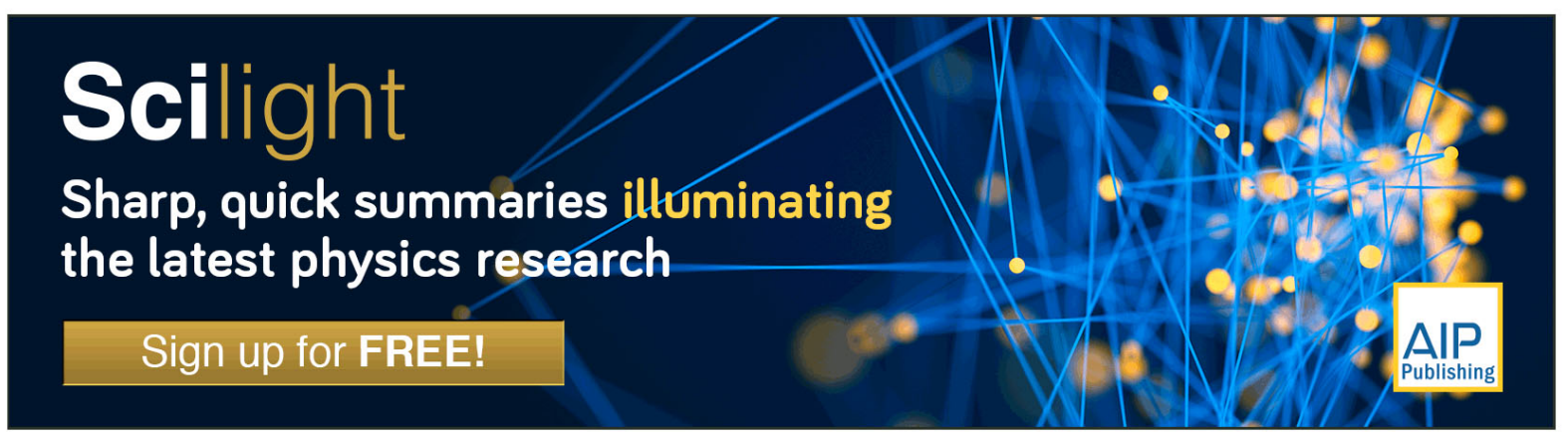




\title{
A versatile atomic force microscope integrated with a scanning electron microscope
}

\author{
J. Kreith, ${ }^{1,2}$ T. Strunz, ${ }^{3}$ E. J. Fantner, ${ }^{3}$ G. E. Fantner, ${ }^{4}$ and M. J. Cordill ${ }^{2}$ \\ ${ }^{1}$ Department of Material Physics, Montanuniversität Leoben, Jahnstrasse 12, 8700 Leoben, Austria \\ ${ }^{2}$ Erich Schmid Institute of Materials Science, Austrian Academy of Sciences, Jahnstrasse 12, \\ Leoben 8700, Austria \\ ${ }^{3}$ GETec Microscopy GmbH, Vienna, Austria \\ ${ }^{4}$ Laboratoray for Bio- and Nano-Instrumentation, EPFL, Lausanne, Switzerland
}

(Received 23 February 2017; accepted 28 April 2017; published online 17 May 2017)

\begin{abstract}
A versatile atomic force microscope (AFM), which can be installed in a scanning electron microscope (SEM), is introduced. The flexible design of the instrument enables correlated analysis for different experimental configurations, such as AFM imaging directly after nanoindentation in vacuum. In order to demonstrate the capabilities of the specially designed AFM installed inside a SEM, slip steps emanating around nanoindents in single crystalline brass were examined. This example showcases how the combination of AFM and SEM imaging can be utilized for quantitative dislocation analysis through the measurement of the slip step heights without the hindrance of oxide formation. Finally, an in situ nanoindentation technique is introduced, illustrating the use of AFM imaging during indentation experiments to examine plastic deformation occurring under the indenter tip. The mechanical indentation data are correlated to the SEM and AFM images to estimate the number of dislocations emitted to the surface. Published by AIP Publishing. [http://dx.doi.org/10.1063/1.4983317]
\end{abstract}

\section{INTRODUCTION}

Several years after the scanning tunneling microscope and the atomic force microscope (AFM) ${ }^{1}$ had been invented, researchers considered to use AFMs inside scanning electron microscopes (SEMs) in order to combine the complementary benefits of both imaging techniques. Some of the reasons include the fact that SEMs provide images with large scan areas up to a few square millimeters at high scan rates, while AFMs provide a high resolution surface topography down to the atomic scale and are able to quantitatively characterize the height of surface features. AFM devices for use in the SEM with different designs, geometries, and various methods for detecting the cantilever deflection signal inside the SEM have already been developed and commercial systems are available..$^{2-4}$ Depending on the design of the according instruments, spatial resolutions of the AFM down to the atomic scale are possible to quantify surface features such as slip steps. ${ }^{5}$

Mechanical testing at small length scales is a popular area of interest in materials research used to investigate size dependent mechanical properties. The availability of focused ion beam (FIB) milling devices, nanoindenters, and dedicated micro- and nano-mechanical testing devices has opened a broad field of research in this area. The SEM is a standard investigation tool for characterization during and after testing. ${ }^{6,7}$ In order to get a high resolution surface topography of the tested macroscopic samples, AFMs have been used independently of the SEM as ex situ and in situ instruments. ${ }^{4-8}$ More recently, AFMs have also been utilized for in situ mechanical testing. For example, deformation analyses and crack propagation with 3- and 4-point bending $9-11$ and bulge testing, ${ }^{12,13}$ as well as buckle delamination and deformation of thin films on polymer substrates, ${ }^{14,15}$ have employed AFM to observe and quantify mechanical behavior during discrete deformation steps.

Complimentary to the SEM as a standard tool for micromechanical experiments, AFMs can also contribute to investigations of mechanically deformed micro-sized compression pillars or bending beams as well as indent imprints made into bulk metals. For example, the heights of slip steps formed during indentation of single crystalline metals can be measured and related to the emitted dislocations. ${ }^{5}$ Slip step heights are typically in the range of $0.1 \mathrm{~nm}$ to $200 \mathrm{~nm}$, and are difficult to quantitatively measure, even using stateof-art SEMs. To optimize these investigations, they should be performed in vacuum in order to reduce the effects of oxide formation that can obscure slip steps. Using AFM, Nibur et al. found that the addition of hydrogen increased the slip step heights and spacing in coarse grained stainless steels. $^{5}$

Combined AFM/SEM devices already exist, but they do not meet the requirements for in situ nanoindentation or micro-mechanical experiments with regard to the required flexibility of the system to keep the AFM and an indenter within a SEM-chamber. The main goal of this work was to develop a setup using a combined AFM/SEM instrument $\left(\mathrm{AFSEM}^{\mathrm{TM}}\right.$ ) that is compatible with in situ micro- and picoindenters that are designed for use in the SEM. The in situ micro- and pico-indenters often have limitations that restrict their use in combination with commercially available AFM/SEM combinations. Examples of the new AFSEM capabilities will be shown using slip steps, the traces of plastic deformation on surfaces around nanoindentation imprints, and a new nanoindentation approach called "Indent@edge" to demonstrate the compatibility of the AFSEM with existing 
in situ indenters for micro-mechanical testing. The mechanical data obtained from the nanoindenter will be correlated to the SEM and AFM images to provide more insight into how plastic deformation occurs under indentation loading.

\section{INSTRUMENTATION}

The AFSEM instrument, developed by GETec Microscopy GmbH (Vienna, Austria), uses piezo-resistive, self-sensing cantilevers. ${ }^{16-18}$ In comparison, commercially available AFMs for the SEM typically use an optical lever ${ }^{19}$ or tuning fork geometry. ${ }^{20}$ The actuation and positioning of the cantilever are performed by piezoelectric ceramic multilayer actuators. For the positioning of the cantilever, a set of two actuators per degree of freedom, mounted on a flexure-based ${ }^{21}$ aluminum frame, is used. Additionally, a single multi-layer piezo actuator excites the cantilever to oscillate in tapping mode. The controller and the software for the AFM instrument are provided by Anfatec Instruments AG (Oelsnitz, Germany). The high voltage amplifier driving the multilayer actuators was built by Techproject (Vienna, Austria).

AFM scanning was carried out in the vacuum of the SEM chamber, using a scanning speed of 0.3 lines/s. The scanning speed in vacuum is limited by the Q-factor (quality factor) of the cantilever ${ }^{22}$ and vibrations of the system. Two main sources of vibrations should be mentioned: external sources like footfall or vibrations of the building and internal sources of the SEM such as the turbo pump or cooling fans of the electronic components. Depending on the required z-resolution, the AFM's sensitivity to noise and accordingly the demand for vibration damping can be considerably higher compared to the requirements for SEM.

To be able to use a wide range of in situ testing equipment and sample geometries for a variety of experiments, the AFSEM unit is able to move independently of the sample stage. It is mounted on a custom built coarse positioning stage providing three translational degrees of freedom and is attached to the SEM chamber door. The sample or testing device for in situ experiments is mounted on the sample stage of the SEM (Figure 1). The setup shown in Figure 1(b) consists of a Zeiss Leo 982 SEM and a Hysitron PI85 pico-indenter ${ }^{23}$ (Minneapolis, Minnesota, USA).

The coarse positioning stage is driven by vacuum compatible stepper motors from Phytron GmbH (Groebenzell, Germany) in the $y$ - and z-directions (the axes directions are shown in Figure 1(a)). These axes are used for parking, deploying, and for coarse approach of the AFM to the sample surface. The $\mathrm{x}$-motor, which is used to align the AFSEM with the SEM electron beam, is a piezo stick-slip motor from PI instruments $\mathrm{GmbH} \& \mathrm{Co}$. KG (Karlsruhe, Germany). An important attribute of the instrument is that after mounting the sample and the cantilever, steps for the adjustment, alignment, and positioning of all of the parts of the setup can easily be performed remotely after evacuating the SEM system from the outside of the chamber using a computer software. The AFM has a scan range of $35 \times 35 \times 8 \mu \mathrm{m}$, the typical scanning speeds in air are 1 line/s to 5 lines/s and in vacuum 0.25 lines/s to 1 lines/s. The maximum sample size of the presented setup is $50 \times 50 \times 80 \mathrm{~mm}$, however, it depends on the type of SEM-chamber used. The geometry of the AFSEM housing allows for an SEM working distance of 6-7 mm, which is relatively small and needed for high resolution SEMimages. The AFSEM is also suitable for measurements in air.

\section{EXPERIMENTAL}

The capabilities of the AFSEM are demonstrated with two experiments: First, images of slip steps on an indented $\mathrm{Cu} 15$ $\mathrm{Zn}$ (brass) single crystal surface and second, the plastically deformed surface of the same material after applying a new in situ nanoindentation testing method, called Indent@edge. The first method provides a lateral and the second method vertical information about the size and nature of the plastic zone evolving around indents.

For both examples, the same $\{111\}$ single crystal brass surface was prepared for indentation by mechanically grinding and polishing, which was followed by electrolytic polishing to remove the residual plastically deformed surface layer to reveal the undamaged single crystalline surface. ${ }^{24,25}$ Directly after the surface preparation, indents with different loads were made with a Hysitron TriboScope nanoindenter equipped with a Berkovich indenter tip $(\mathrm{R}=750 \mathrm{~nm})$. Immediately after indenting, the sample was put into the SEM. By keeping the times in air as short as possible, the negative effects due to oxidation of the sample surface were minimized.

The second example consisted of a well-defined $90^{\circ}$ edge made using the same brass single crystal. Similar to the preparation process above, after mechanically cutting, grinding, and polishing on both adjacent surfaces, the plastically deformed surface layer was removed by electrolytic polishing. As a final step in order to cope with edge rounding caused by the electrolytic polishing, one face was additionally ion polished using a Hitachi E-3500 ion milling system. In the SEM, one
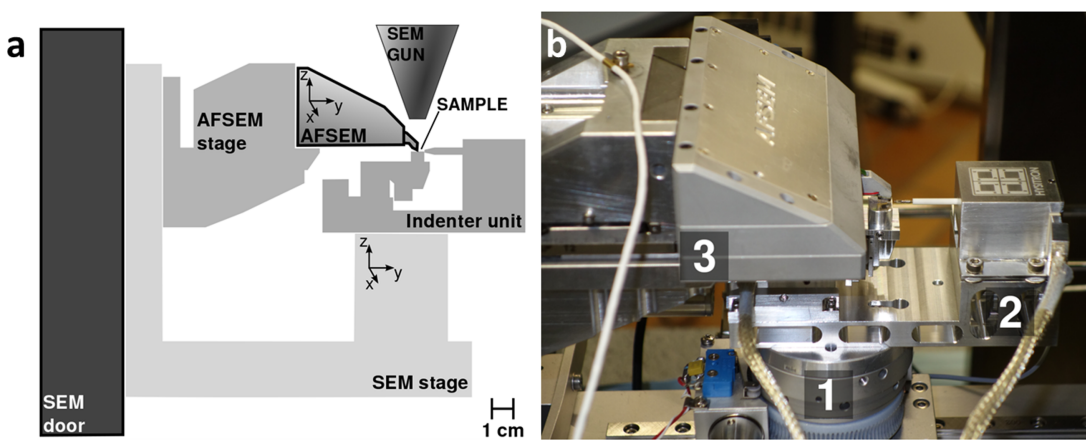

FIG. 1. (a) Schematic diagram of the in situ micro-mechanical testing setup with AFSEM on the door of the SEM and the indenter unit mounted on the SEM sample stage. (b) Photo of the opened SEM chamber with SEM sample stage (1), indenter unit (2), and $\operatorname{AFSEM}(3)$. 
a

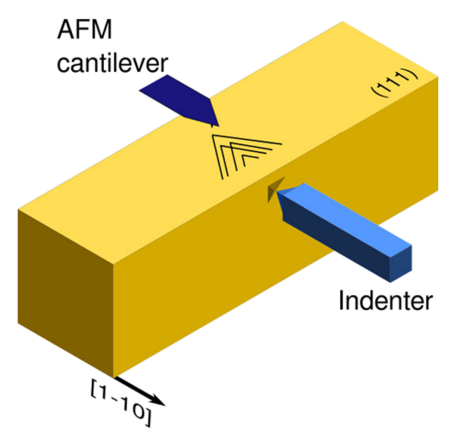

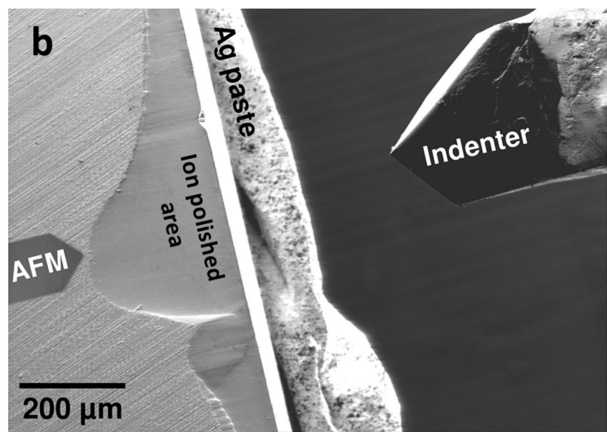

FIG. 2. (a) Schematic diagram of the Indent@edge setup. The sample is indented close to the polished edge while the adjacent surface is imaged by the AFM. (b) SEM overview of the Indent@edge setup. The top surface of a $90^{\circ}$ edge is investigated by the AFM (cantilever partially seen on the left) after the adjacent surface (orthogonal to the image plane, visible as diagonal bright streak in the left third of the image) was indented close to the top surface by a cube corner indenter tip (seen on the top right corner).

side of the freshly prepared $90^{\circ}$ corner was indented close to the top edge (approximately $500 \mathrm{~nm}$ ) using a Hysitron PI85 Picoindenter with a cube corner tip $(\mathrm{R}=270 \mathrm{~nm})$ after which the adjacent surface was imaged with the AFM to record evolving slip steps providing information of the plastically deformed zone under the indent. A schematic diagram of the Indent@edge setup is shown in Figure 2. For all of the presented experiments, a Zeiss Leo 982 large chamber SEM was used to repeatedly find certain areas of interest on the sample for AFM imaging. ${ }^{2,26}$ To minimize sample contamination caused by hydrocarbon residues due to the electron beam interaction, ${ }^{27}$ the SEM imaging times during the AFM cantilever approach to the sample were kept as short as possible and the AFM scans were always performed before high resolution SEM imaging.

\section{RESULTS}

In the first example, nanoindentation of the brass single crystal caused slip steps to appear at the surface. The loaddisplacement curve of a $3 \mathrm{mN}$ indent created with the Hysitron TriboScope is shown in Figure 3(a). The surface is first loaded elastically until plastic deformation is initiated in the form of a spontaneous collective movement of dislocations, indicated by the pop-in event (also referred to as an excursion in the literature ${ }^{28,29}$ ). After the pop-in event, the plastic deformation proceeds continuously until the maximum pre-defined load is reached. The hysteresis of the loading curve is a measure of the total plastic deformation of the surface. The SEM was necessary to find the nanoindents for AFM imaging because of the small size of the residual imprints. The first indent leaving a large permanent imprint in the brass surface visible with an optical light microscope which required a load of $500 \mu \mathrm{N}$ was easily located in the SEM. Another imaging method would be to use the nanoindenter tip right after the indentation experiment; however, the large radius of the indenter tip causes a poor image resolution and inaccurate height measurement. Figures 3(b) and 3(c) contain images of slip steps which are formed around the indent, recorded by the SEM and the new in situ AFM. It is important to note that the SEM image (Figure 3(b)) does not show the same amount of detail as the AFM image (Figure 3(c)). AFM is the best available technique that can accurately image indent imprints and measure slip step heights on the required scale. From the SEM image in Figure 3(b), the indent imprint is clearly observed and slip steps can be viewed only at the indent edges. The particle to the left is the same as in the AFM image (Figure 3(c)). More surface details are visualized and measureable in the AFM image due to the additional height information.

The new Indent@edge technique was used to demonstrate that in situ nanoindentation experiments are possible with the available in situ indenter and AFSEM. The surface close to a $90^{\circ}$ edge of a single crystalline brass sample is indented and the adjacent side is imaged by the AFM to record the plastically deformed surface. The indentation experiment was carried out by several consecutive loading
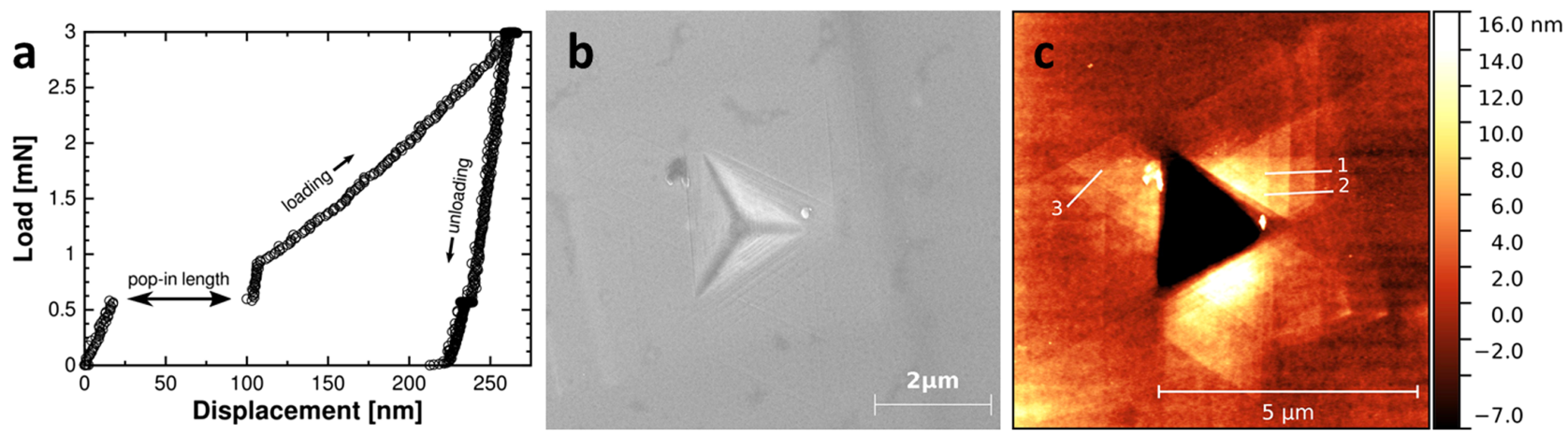

FIG. 3. (a) Load-displacement curve of the $3 \mathrm{mN}$ indent illustrating a pop-in event. (b) SEM image of $3 \mathrm{mN}$ Berkovich nanoindent on a single crystalline brass sample surface and (c) AFM image of the same indent as shown in (b). Profiles marked in (c) are shown in Figure 5(b). 

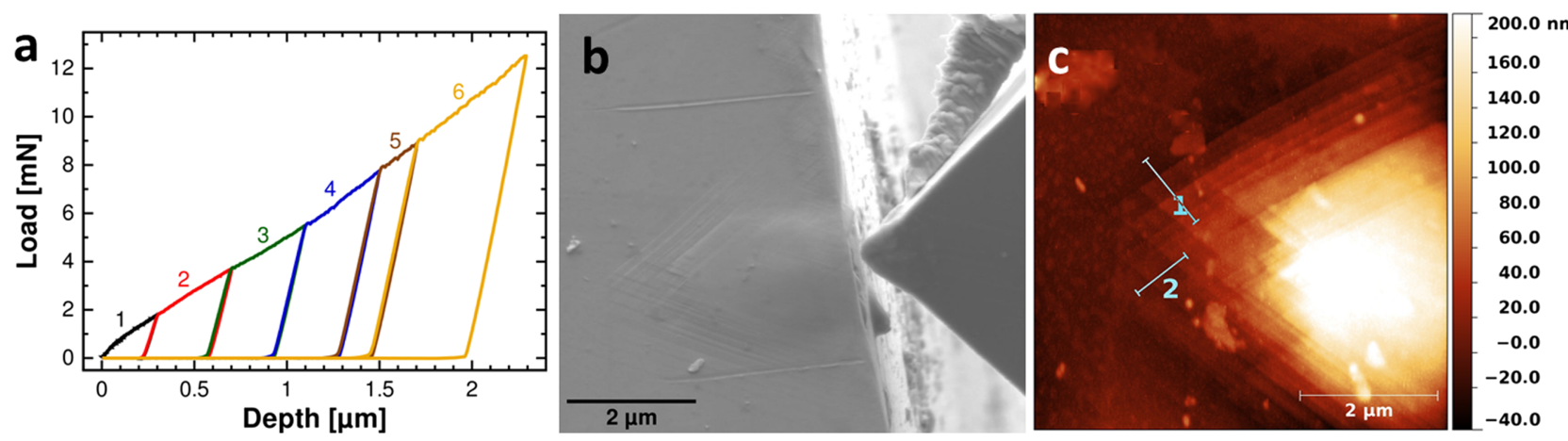

FIG. 4. (a) Load-displacement curve of the Indent@edge experiment. The experiment was carried out in six successive loading steps with only the last loading step resulting in slip step formation under the indenter tip. (b) SEM overview after the experiment, showing the indenter tip (right) and the plastically deformed sample surface (left). (c) AFM overview of the plastically deformed zone after the Indent@edge experiment.

steps at the same sample position (Figure 4(a)). After each loading step, the maximum indentation load was increased until the plastic deformation of the surface in the form of slip steps was observed by the SEM (Figure 4(b)) which occurred only on the last loading step (max. load $12.5 \mathrm{mN}$ ). The plastically deformed region was scanned by the AFM (Figure 4(c)) to analyze the size of the plastic zone and to estimate the number of emitted dislocations by extracting the 1D profiles. During the Indent@edge experiment, no pop-in events were observed. The indentation position close to the edge is less constrained for a pop-in to occur compared to the conventional indentation experiment and a sharper tip was used ( $270 \mathrm{~nm}$ cube corner). With the smaller tip radius, fewer statistically stored dislocations are activated during the indentation process.

\section{DISCUSSION}

With the additional height information provided by the AFM images, the amount of plasticity induced by indentation can be quantified using the slip step heights. As shown in the AFM image of surface around the $3 \mathrm{mN}$ indent (Figure 3(c)), the angles between the slip lines are $60^{\circ}$ and strictly correlate to the (110) crystal directions. This indicates that the surface of the FCC brass single crystal is of type $\{111\}^{30}$ and was confirmed by electron backscatter diffraction. With the knowledge of the crystal directions and the orientation of the sample surface, the active slip systems can be identified. This makes it possible to determine the number of dislocations which have been emitted to the surface (Figure 5(a)). The direction of the active slip system $(\vec{b})$, which corresponds to an energetically favored direction in the crystal system, is projected to the unit normal vector $(\hat{n})$ of the sample surface. The number of emitted dislocations, $n_{d}$, is obtained by dividing the perpendicular distance, $|\vec{n}|$, by the projected distance of a single dislocation which corresponds to the length of the Burgers ${ }^{31}$ vector, giving $n_{d}=|\vec{n}| /(\vec{b} \cdot \hat{n})$. The possible slip systems in FCC crystals are the $<110>$ directions on the $\{111\}$ crystal planes. The length of the Burgers vector, $b$, is the distance of two adjacent atoms in the $<110>$ direction giving

$$
b=\frac{a}{2}|(110)|,
$$

where $a$ is the lattice constant of the material. For the brass sample, the lattice constant was determined with X-ray diffraction and resulted in $3.65 \AA$. Therefore, the length of the Burgers
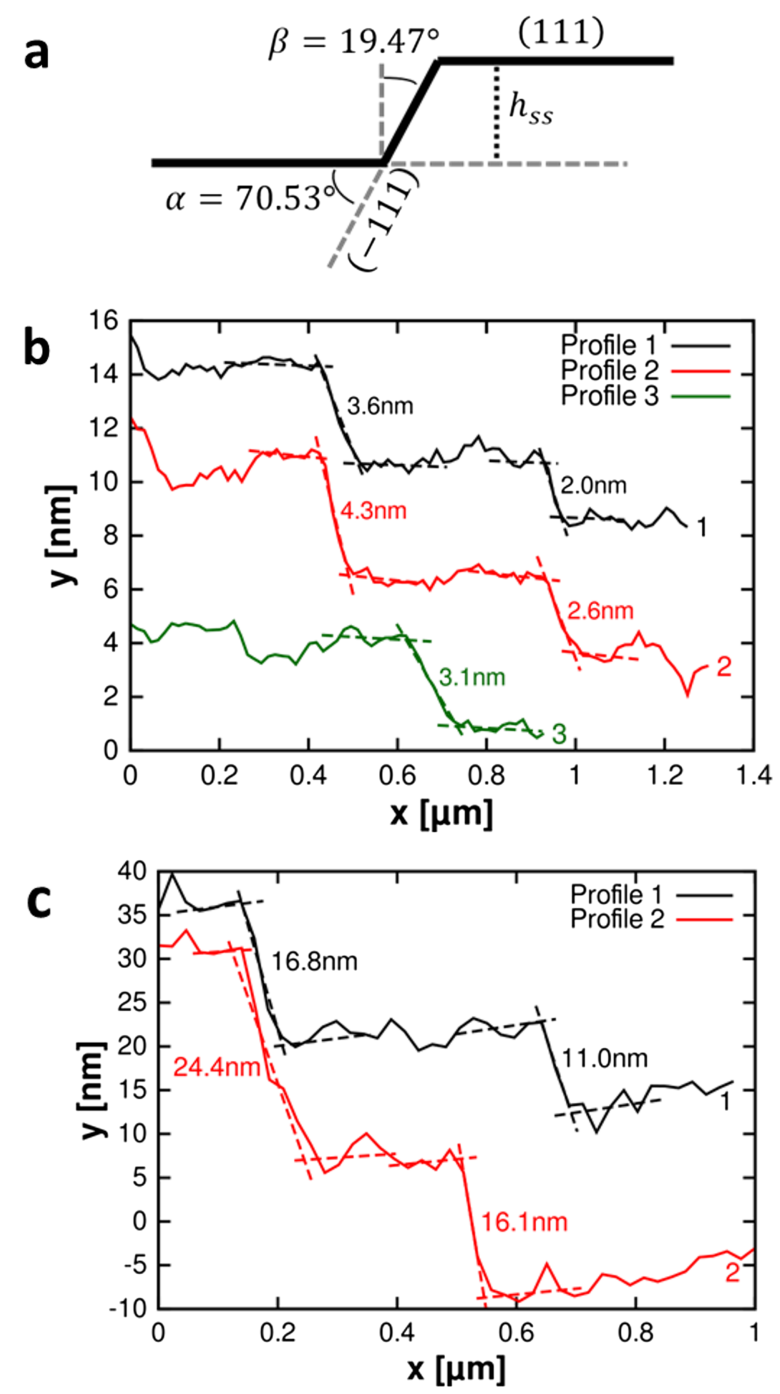

FIG. 5. (a) Schematic diagram of the slip step geometry and measurement. (b) $1 \mathrm{D}$ profiles at the positions indicated in Figure 3(c). To measure the height of the slip steps, the profile data close to the steps were fitted to lines by assuming the same slope on the left and right side of the step (dashed lines). The perpendicular distance of the fit lines is inserted into the graph. (c) 1D profiles as indicated in Figure 4(c) of the Indent@edge experiment. 
vector is $2.58 \AA$. The vertical height of the slip step, $h_{s}$, is related to the number of emitted dislocations using

$$
n_{d}=\frac{\sqrt{3 / 2}}{b} h_{s s} .
$$

As an example, the profiles indicated in Figure 3(c) are plotted in Figure 5(b). The profile data close to a slip step is fitted to two separate lines at the left and right side of the step in order to determine the slip step heights. By assuming that the creation of the slip step locally does not alter the curvature of the surface, the slope of the two fit lines has to be equal. The perpendicular distance of the two parallel fit lines is evaluated using the fit parameters and is the measured slip step height, $h_{s s}$. In Figure 5(b), the slip step heights range between $2 \mathrm{~nm}$ and $4 \mathrm{~nm}$ and correlate to $10-20$ dislocations being emitted to create the slip step. The error of the height measurement is in the range of $0.2 \mathrm{~nm}$ to $0.3 \mathrm{~nm}$ equivalent to one to two dislocations. The data range of the lines fitted to the slip steps must be chosen carefully. Besides the $3 \mathrm{mN}$ indent shown here, a series of indents in the load range of $50 \mu \mathrm{N}$ to $10 \mathrm{mN}$ was also investigated. For all indents, the height of the slip steps is in a similar range of less than $10 \mathrm{~nm}$. These results follow the model of Nibur et al. ${ }^{5}$ which states that the size of the plastic zone around the indent continues to expand with increasing loads by forming new slip steps in favor of increasing the height of the already existing ones.

The same evaluation method was used to evaluate the number of emitted dislocations from the Indent@edge example (Figure 5(c)). Slip step heights of $10 \mathrm{~nm}$ to $25 \mathrm{~nm}$ were observed to form under the indent. These slip step heights are larger than what was found for the slip steps that form around indents on the surface. The number of dislocations emitted to the surface under the indent was determined to be between 52 and 116 dislocations. Compared to the dislocations that reach the top surface, 5 times more dislocations are observed under the indent in the Indent@edge configuration. This is most likely due to the fact that in order for dislocations to reach the top surface (i.e., $3 \mathrm{mN}$ indent example, Figure 3), dislocations must cross-slip, while for the Indent@edge example, the dislocations only need to glide to the free surface. ${ }^{32-34}$

The Indent@edge method is an interesting and a novel way to examine nanoindentation deformation. It must be noted that because an indent is made near the edge, the mechanical behavior (load-displacement curve) will not provide the same behavior as an indent performed in the bulk material. An example is to compare Figure 3(a) with Figure 4(a), but recall that two different indenter tips were also used. In the bulk, pop-ins were observed (Figure 3(a)) and at the edge, no pop-ins (Figure 4(a)). This is due to the fact that there is less constraint around the indent near an edge. As most nanoindentation researchers know, it is not good scientific practice to indent in close proximity to an edge, thus, edges are avoided. The Indent@edge method can still provide worthwhile information even without meaningful load-displacement curves. As demonstrated, the slip step type and quantifiable dislocation information were obtained. The plastic zone size under the indent can be quantified with this method, direct examination of grain growth or grain boundary sliding under indents, and even fracture events in ceramics or thin films could be investigated with Indent@edge. The sample preparation could be a limitation if a research lab does not have a means to fabricate samples with a $90^{\circ}$ edge. Furthermore, a wedge indenter, often used for in situ TEM indentation experiments, could be used instead of a Berkovich or cube corner geometry and would deliver a more accurate look at the deformation forming under the indenter without worrying about how close one is to the edge.

\section{SUMMARY}

An AFM has been successfully incorporated into an SEM for use in combination with in situ nanoindentation testing experiments. The SEM stage is used as the sample holder and the AFM unit is positioned independently from the SEM sample stage using a motorized 3D stage. Slip steps emanating from nanoindentation imprints on single crystal brass were measured to estimate the number of dislocations which reach the surface, and first in situ experiments with an in situ nanoindenter (Indent@edge) were performed using AFSEM. The SEM is needed to aid in positioning the AFM to the sub-2 $\mu \mathrm{m}$ sized features and the AFM images allow for the quantification of the deformation in single crystals (slip steps) which occur in the z-direction (out-of-plane). Single slip steps having dimensions of at least a nanometer can be resolved by fitting the height data. Using the height of slip steps, the number of emitted dislocations was quantified for dislocations which cross-slip to the surface and those that do not need to cross-slip. It was found that less than half the number of dislocations reach the surface when they must cross-slip compared to dislocations which do not cross-slip. By using the AFSEM, the indentation experiment and the AFM scan take place in vacuum so that contamination or oxidation of the sample surface caused by ambient air during an in situ experiment is minimized. The success of the Indent@edge approach further proves that it is possible to perform in situ micro-mechanical experiments such as micro pillar compression or beam bending and to quantify the dislocation activity in small volumes without the risk of sample contamination. The success of these experiments illustrates the importance that the combined AFM/SEM imaging provides nanoindentation as well as future micro-mechanical experiments which would benefit from simultaneous AFM and SEM imaging.

\section{ACKNOWLEDGMENTS}

Funding for this research has been provided by the Austrian Research Promotion Agency (FFG) under Project No. 829639, from the European Union FP7/2007-2013/ERC under Grant Agreement No. 307338-NaMic, and EUROSTARS CoD10 8213 TRIPLE-S Microscope.

\footnotetext{
${ }^{1}$ G. Binnig, C. Quate, and C. Gerber, Phys. Rev. Lett. 56, 930 (1986).

${ }^{2}$ A. V. Ermakov and E. L. Garfunkel, Rev. Sci. Instrum. 65, 2853 (1994).

${ }^{3}$ S. Bauerdick, C. Burkhardt, R. Rudorf, W. Barth, V. Bucher, and W. Nisch, Microelectron. Eng. 67-68, 963 (2003).

${ }^{4}$ W. F. Oele, J. W. J. Kerssemakers, and J. T. M. De Hosson, Rev. Sci. Instrum. 68, 4492 (1997).

${ }^{5}$ K. A. Nibur, F. Akasheh, and D. F. Bahr, J. Mater. Sci. 42, 889 (2007).
} 
${ }^{6}$ M. D. Uchic, D. M. Dimiduk, J. N. Florando, and W. D. Nix, Science 305, 986 (2004).

${ }^{7}$ D. Kiener, W. Grosinger, G. Dehm, and R. Pippan, Acta Mater. 56, 580 (2008).

${ }^{8}$ M. J. Cordill and V. M. Marx, Philos. Mag. Lett. 93, 618 (2013).

${ }^{9}$ M. Göken, Rev. Sci. Instrum. 65, 2252 (1994).

${ }^{10}$ M. Göken, H. Vehoff, and P. Neumann, J. Vac. Sci. Technol., B: Microelectron. Nanometer Struct. 14, 1157 (1996).

${ }^{11}$ M. Göken, H. Vehoff, and P. Neumann, Scr. Metall. Mater. 33, 1187 (1995).

${ }^{12}$ E. W. Schweitzer and M. Göken, J. Mater. Res. 22, 2902 (2011).

${ }^{13}$ B. Merle and M. Göken, J. Mater. Res. 29, 267 (2014).

${ }^{14}$ V. M. Marx, C. Kirchlechner, I. Zizak, M. J. Cordill, and G. Dehm, Philos. Mag. 95, 1982 (2015).

${ }^{15}$ M. J. Cordill, O. Glushko, J. Kreith, V. M. Marx, and C. Kirchlechner, Microelectron. Eng. 137, 96 (2014).

${ }^{16}$ G. E. Fantner, D. J. Burns, A. M. Belcher, I. W. Rangelow, and K. Youcef-Toumi, J. Dyn. Syst., Meas., Control 131, 61104 (2009).

${ }^{17}$ G. E. Fantner, W. Schumann, R. J. Barbero, A. Deutschinger, V. Todorov, D. S. Gray, A. M. Belcher, I. W. Rangelow, and K. Youcef-Toumi, Nanotechnology 20, 434003 (2009).

${ }^{18}$ M. Dukic, J. D. Adams, and G. E. Fantner, Sci. Rep. 5, 16393 (2015).

${ }^{19}$ G. Meyer and N. M. Amer, Appl. Phys. Lett. 53, 1045 (1988).

${ }^{20}$ P. Guenther, U. C. Fischer, and K. Dransfeld, Appl. Phys. B: Photophys. Laser Chem. 48, 89 (1989).
${ }^{21}$ G. E. Fantner, G. Schitter, J. H. Kindt, T. Ivanov, K. Ivanova, R. Patel, N. Holten-Andersen, J. Adams, P. J. Thurner, I. W. Rangelow, and P. K. Hansma, Ultramicroscopy 106, 881 (2006).

${ }^{22}$ T. R. Rodríguez and R. García, Appl. Phys. Lett. 82, 4821 (2003).

${ }^{23}$ D. S. Gianola, A. Sedlmayr, R. Mönig, C. A. Volkert, R. C. Major, E. Cyrankowski, S. A. S. Asif, O. L. Warren, and O. Kraft, Rev. Sci. Instrum. 82, 063901 (2011).

${ }^{24}$ W. C. Oliver and G. M. Pharr, J. Mater. Res. 7, 1564 (1992).

${ }^{25}$ Z. Wang, H. Bei, E. P. George, and G. M. Pharr, Scr. Mater. 65, 469 (2011).

${ }^{26}$ U. Stahl, C. W. Yuan, A. L. De Lozanne, and M. Tortonese, Appl. Phys. Lett. 65, 2878 (1994).

${ }^{27}$ A. E. Vladar, M. T. Postek, and R. Vane, Proc. SPIE 4344, 835-843 (2001).

${ }^{28}$ M. J. Cordill, M. S. Lund, J. Parker, C. Leighton, A. K. Nair, D. Farkas, N. R. Moody, and W. W. Gerberich, Int. J. Plast. 25, 2045 (2009).

${ }^{29}$ M. J. Cordill, N. R. Moody, and W. W. Gerberich, J. Mater. Res. 23, 1604 (2008).

${ }^{30}$ S. C. Chang and H. C. Chen, Acta Metall. Mater. 43, 2501 (1995).

${ }^{31}$ J. M. Burgers, Proc. K. Ned. Akad. Wet. 42, $293(1939)$.

${ }^{32}$ M. Rester, C. Motz, and R. Pippan, Acta Mater. 55, 6427 (2007).

${ }^{33}$ W. W. Gerberich, J. C. Nelson, E. T. Lilleodden, P. Anderson, and J. T. Wyrobek, Acta Mater. 44, 3585 (1995).

${ }^{34}$ W. W. Gerberich, M. J. Cordill, W. M. Mook, N. R. Moody, C. R. Perrey, C. B. Carter, R. Mukherjee, and S. L. Girshick, Acta Mater. 53, 2215 (2005) 